Annales d'Éthiopie, 2000, vol. XVI: 93-106.

\title{
REPRÉSENTATIONS dE LA CULTURE MATÉRIELLE DES ME'EN : FORME ET FONCTION DES ARTEFACTS ${ }^{1}$
}

\author{
Jon ABBINK*
}

Résumé : Cette étude présente une première analyse de la culture matérielle des $\mathrm{Me}$ 'en (Tishana), une ethnie de l'Éthiopie méridionale. Il s'agit d'une société sans artisans ou artistes spécialisés. La question envisagée est de savoir s'il existe des critères esthétiques sous-jacents au sein d'une culture matérielle comprenant pour l'essentiel des objets d'usage quotidien, avec la perspective de dénier, dans le domaine de l'étude comparative des cultures matérielles, la distinction entre culture de "grand art" et culture d'art "rudimentaire".

Mots-clefs : Me'en, esthétique, culture matérielle, arts "ethniques"

Summary : This article presents a tentative analysis of the material culture of the Me'en people of Southern Ethiopia, a society without specialized artisans or artists. The question is to discover underlying criteria of aesthetics in a tradition of material culture that mostly consists of "artefacts of daily use" and not of high art. The ultimate aim hereby is to disclaim the analytic and evaluative distinction between "high" art and "simple" art in the comparative study of material culture.

Keywords : Me'en people, aesthetics, material culture, "ethnic" arts.
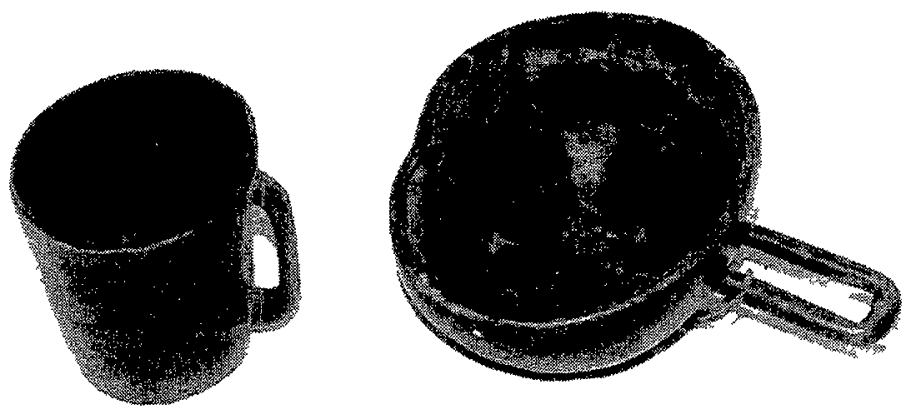

Photo 1 (J Abbink).

\footnotetext{
* Centre d'Études Africaines de Leiden.

${ }^{1}$ Cet article est une version très adaptée d'un chapitre écrit il y a trois ans pour un livre (non publié) intitulé Ethiopia : Traditions of Creativity. Ce livre devrait accompagner l'exposition du même nom qui s'est tenue au Musée de Michigan State University (East Lansing, USA) en 1994. Les recherches de terrain et la collecte des objets me'en pour le musée ont été réalisées dans le cadre de ce projet, dirigé par le Professeur Raymond W. Silverman.
} 
Est-ce que toutes les sociétés connaissent l'art, une tradition artistique ou, du moins, des "artistes"? Je pose cette question à propos de l'étude de la culture matérielle des $\mathrm{Me}$ 'en, une ethnie de l'Éthiopie méridionale constituée d'anciens pasteurs nomades actuellement devenus des cultivateurs plus ou moins sédentaires. Lors de mes recherches de terrain, effectuées au début des années 90 , il m'était difficile de réprimer un sentiment de déception en constatant chez eux une tradition esthétique très "simple". Un tel biais subjectif a caractérisé la description et l'interprétation de beaucoup de traditions non-occidentales en général, à l'exception des traditions chrétiennes et islamiques de l'Éthiopie des hauts plateaux. Cependant, les Me'en semblaient bien posséder des critères d'esthétique, bien que leurs objets soient de petite taille et guère "spectaculaires". Le présent défi consiste à présenter et à expliquer ces critères, en reconsidérant le paradigme dominant dans le domaine des études sur l'art non-occ1dental.

En descendant les hauts plateaux éthiopiens du centre et de l'est, caractérisés par de riches traditions chrétiennes et islamiques (Tegray, Bägémder, Choa, Wällo, Jimma ou Harar), vers l'ethnie des Me'en, qui habite une région assez éloignée au sud-ouest, on ne semble pas rencontrer une culture matérielle très développée ${ }^{2}$. Les Me'en, ce groupe de cultivateurs et d'éleveurs de bétail, ne possèdent pas une classe spécialisée d'artistes ou d'artisans reconnue. Ils ne produisent pas cet art remarquable, figuratif ou rituel, que l'on peut trouver ailleurs en Afrique centrale ou occidentale, ou même dans quelques régions de l'Éthiopie, et qui est caractérisé par les masques, les peintures, l'architecture religieuse, les images de bois ou les paniers colorés.

Les premières questions qui me semblent devoir être formulées face à une culture matérielle dite relativement simple sont les suivantes: quand et comment pouvonsnous identifier une "tradition artistique" quand elle n'est pas reconnue comme telle au sein de la société (par exemple, en l'absence d'artistes spécialisés) ? Les producteurs ou les artisans font-ils référence aux idéaux de "beauté" des objets matériels qu'ils produisent, même quand il s'agit d'objets "utilitaires" ? Ces objets eux-mêmes possèdentils - en plus de leur fonction d'objet domestique, d'outil, ou de décoration - des référents sémantiques ou symboliques ? Autrement dit, le monde des objets est-il partout un domaine de symbolisme et de valorisation culturelle ?

Or nous savons que les peuples, en Afrique ou ailleurs, ne relèvent pas des mêmes définitions de "l'art" et de la "beauté" : évidemment, ces notions sont pour l'essentiel partie intégrante d'un discours et d'une sensibilité occidentale à l'esthétisme, souvent détachée de la vie quotidienne (GELL 1992). Ce qui signifie que l'idée d'un art autonome constitue une catégorie culturelle spécifique. Certes la discussion n'est pas nouvelle. Au cours des années 80 , il y a eu des débats portant sur la distinction art / artefacts dans la littérature africaniste (KLOPPER 1993: 39). Ethnologues et spécialistes d'art "tribal" ou "traditionnel" ont souvent souligné qu'on se devait de prendre connaissance du contexte culturel et de la trajectoire historique complète d'une tradition d'artefacts. La production, la distribution et l'utilisation des artefacts se distin-

${ }^{2}$ Pour une étude des objets d'une autre société éthiopienne "simple" (du point de vue technologique), vorr" TORNAY 1975 L'auteur donne une analyse valable du contexte social et économique de la culture matérelle des agro-pasteurs Nyangatom. 


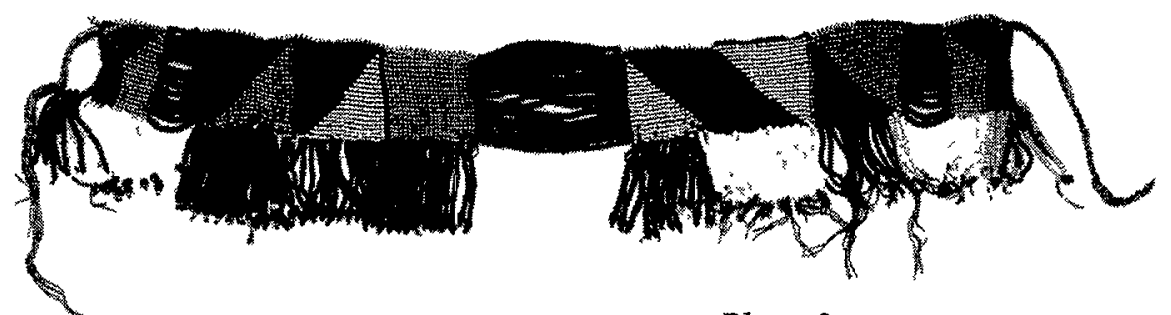

Photo 2 (J. Abbink).

guent par des éléments fonctionnels aussi bien que sociaux; on ne peut pas les isoler et les fixer comme des phénomènes artistiques détachés. De plus, il est difficile de les évaluer a priori avec des critères externes, surtout issus de la sensibilité occidentale, et qui concernent la "beauté" et le "chef-d'œuvre".

Mais n'est-il pas possible de cerner au moins le sens esthétique ou artistique qui se révèle dans un objet quand il se présente à nous ? Par exemple, quelles sont les notions minimales de forme propre, d'harmonie, d'habileté, d'efficacité ou d'effet visuel appliquées aux objets au sein des sociétés qui ont été déclarées être de tradition esthétique pauvre? On pourrait aussi mettre l'accent sur le producteur et sa relation avec les objets produits, et non pas seulement sur l'objet en tant que tel. Chez les peuples Kasem du Ghana du Nord, on distingue des formes et des qualités de sens artistique chez les producteurs, qui sont détachées des objets (DE CARBO 1977) ${ }^{3}$. On pourrait aussi se rendre compte en premier lieu de la signification acquise par un objet lorsqu'il "vieillit", rassemblant des "histoires" pendant sa circulation, comme dans le cas des objets de kula décrits par MALINOWSKI en 1922.

En faisant des recherches sur les objets me'en, nous avons essayé de formuler des réponses à ces questions à partir d'observations et de discussions avec les producteurs et les utilisateurs. La réponse formulée ici est issue pour une bonne part de ce qu'ils m'ont dit et montré. Tout d'abord, j'ai pris l'exemple de deux producteurs moyens qui produisaient, à ma demande, quelques objets d'art quotidien, comme des calebasses, des paniers, des outils de bois ou des objets de décoration. Je souligne le mot "moyen" : ces hommes n'étaient pas de véritables spécialistes. Quand on leur demande leur profession, ils se présentent d'ailleurs comme des agriculteurs labourant les champs et soignant les vaches, et non pas comme des artistes ou des artisans.

Mes dialogues avec les Me'en m'ont amené à des conclusions préliminaires que je présente ici comme une base pour une discussion plus approfondie. Tout d'abord, il faut être prudent avec l'emploi du terme "art" lorsque l'on interprète les traditions natérielles des sociétés de subsistance non-occidentales et non-lettrées comme celle les Me'en. Chez ceux-ci, l'art est encadré plus profondément par la culture matériele, et est à considérer en premier lieu comme "le processus socio-technologique de rroduction et d'usage d'artefacts", et non pas comme un idéal de beauté autonome. Jeuxièmement, la production des artefacts est à l'évidence un processus social, encaIré par les besoins de la vie quotidienne et les interdépendances sociales des produceurs et des utilisateurs. Troisièmement, même si les objets apparaissent comme

Merci au Professeur Ray Silverman pour cette référence. 
"banals" ou "simples", ou seulement utilitaires, ils relèvent toujours d'une dimension visuelle et d'une "aptitude" de forme et de fonction qui résultent d'une série de cho1x des producteurs concernant la nature et la qualité du matériel, selon les conventions culturelles en vigueur.

Ainsi, un point de vue ethnologique sur la valeur des artefacts me'en tend à souligner non simplement la qualité esthétique attribuée localement à un objet (au sein de la société étudiée) mais en même temps le rôle social qui luı donne sa signification et sa valeur aux yeux des personnes qui en font usage. De plus, on doit se rendre compte de la relation existant entre d'une part les matériaux et les moyens techniques (souvent très limités), et d'autre part le moment de l'intervention personnelle d'un producteur. Autrement dit, la simplicité des moyens techniques et du matériau mis en œuvre n'impliquent pas la simplicité du travail créateur lui-même. Quand l'ethnologue H. AMBORN faisait des recherches chez les orfèvres des Konso et des Burji, 1l essaya lui-même de fabriquer leurs produits. Ayant reçu une formation d'ingénieur, il croyalt ainsi pouvoir comprendre leur travail et être capable de les imiter. Mais il fut forcé d'admettre qu'il ne réussirait jamais à produire un seul objet de fer acceptable (AMBORN 1990: 53).

Ayant ainsi redéfini l'approche de l'art quotidien, il convient de dire que même des objets comme un appui-nuque, une calebasse, un couteau ou une écuelle en bois taillée et polie, se présentent non seulement comme porteurs d'efficacité fonctionnelle et d'aptitude morphologique, mais auss comme transmetteurs de beauté - cela veut dire d'authenticité et d'effort créateur - tout particulièrement dès lors que l'on s'est familiarisé avec les conventions culturelles de la société qui a engendré ces objets, et avec l'histoire de ces mêmes objets parmi les hommes.

En résumé, il faut élargir la catégorie d'art et la définir comme la production technique d'artefacts, la fabrication ou la création humaine d'un objet pour des raisons à la fois esthétiques et utilitarres (GELL 1992 : 43). Les traditions artistiques doivent être comprises comme étant l'une des manifestations de ce processus de réalisation de l'esprit créateur, qui s'exprime dans le domaine des matériaux inanımés en engendrant la culture matérielle.

\section{LE PEUPLE ME'EN}

Les Me'en sont une population rurale de l'Éthiopie méridionale, comptant au moins 58000 personnes environ ${ }^{4}$, et divisée en deux groupes : les Bodi (4 600 indiv1dus) sont des agro-pasteurs avec de grands troupeaux de bétail, qui se situent dans les savanes à l'est du fleuve Omo. Les Tishana (53000) sont des cultivateurs et des chasseurs-cueilleurs au nord et à l'ouest de l'Omo autour des bourgades de Kela et de Jamu. Les deux groupes descendent d'une même population d'agro-pasteurs parlant une langue nilo-saharienne. Ils ont assimilé un bon nombre de petits groupes antérieurs. Les Tishana, par exemple, ont absorbé des Bench et des Dizi (locuteurs de langues omotiques) et aussi des Kwegu (une ethnie établie dans la région du flẹuve

\footnotetext{
${ }^{4}$ Central Statistical Authonty, The 1994 Population and Housing Census of Ethropia Results at the National Level, volume 1, p 66, Addis Ababa CSA
} 
Omo). Les Tishana aussi possèdent du bétail, mais en moins grande quantité que les Bodi. Les deux groupes sont assez mobiles, les derniers en raison des exigences de la transhumance annuelle du bétail, les Tishana - bien que plus sédentarisés - pour chercher de nouveaux champs dans la brousse, tous les deux ou trois ans. Ils n'habitent pas des villages mais des enclos ou des hameaux dispersés.

La plupart des Tishana, moins à l'écart de l'administration que les Bodi, ont été intégrés dans le système des qäbälé (associations de paysans) depuis 1976. En 1993, la réorganisation administrative de l'Éthiopie a créé une "zone" Maji-Bench et un wäräda me'en. La même année, les Me'en étaient forcés par le parti politique au pouvoir, le EPRDF (Ethiopian Peoples' Revolutionary Democratic Front), de former une association ethno-politique me'en qui a été incorporée en 1995 dans un nouveau parti politique rassemblant les Bench, les Dizi, les Sheko et les Me'en, à nouveau sous l'aile de l'EPRDF. L'isolement social et économique des Me'en diminue peu à peu, bien que la plupart des Me'en vivent encore dans une société d'autosubsistance, avec peu d'échanges commerciaux directs avec des non-Me'en.

Les Me'en forment une société assez égalitaire du point de vue socio-économique et politique. Les anciens de clans et de groupes locaux, ainsi que les "maitres de la pluie" nommés komorut, conservent en général le respect du peuple, bien qu'ils n'aient point d'autorité exécutive : ils ne sont pas des "chefs" au sens propre du terme. Il convient de souligner que les Me'en descendent d'une population pastorale typique de l'Afrique de l'Est (voir ABBINK 1990 pour un résumé historique). Les textes nous enseignent que de tels groupes ne se caractérisent pas par une riche tradition de culture matérielle (cf aussi VON GAGERN \& AL. 1974: 38f), et la quantité d'objets produite est de même souvent assez faible. En effet, nous estimons que le nombre d'objets connus chez les Me'en ne dépasse pas le nombre de cent trente. Il faut entendre par là les objets qu'ils produisent eux-mêmes avec des matériaux locaux. En comptant les objets importés depuis les dernières années, on doit en ajouter une vingtaine d'autres (rasoirs, sel, étoffes, coton, savon, souliers, fusils, etc. ; voir ABBINK 1992).

Aujourd'hui, les Tishana-Me'en sont tous des cultivateurs d'autosubsistance, leurs productions principales étant les céréales (sorgho, téf, maïs, froment) et des légumineuses (fèves, haricots et choux verts). Leur bétail est peu nombreux. A part quelques groupes au sud et le groupe Bodi par-delà l'Omo, vers

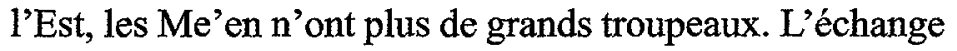
monétaire se fait par la vente du café et surtout du miel. Les marchés régionaux sont peu développés. Le travail agricole est fait par les hommes et les femmes, mais ces dernières travaillent le plus dur. Ils produisent tous les aliments, préparent la bière (sholu) de sorgho et maïs, labourent les champs et les jardins, et aussi vendent les surplus de la famille au marché. Les seuls objets que les femmes produisent sont des poteries (des plats à cuire, en me'en: retech) et des pots pour la bière et le lait (qui se fabriquent en trois tailles : dolé, diskí, et $j u$, le plus grand) utilisés par les Me'en. Comme chez plusieurs ethnies d'Éthiopie méri-

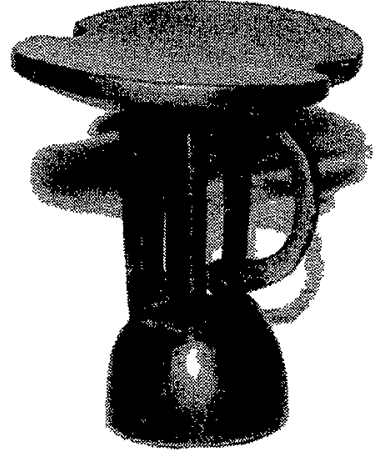

Photo 3 ( $J$ Abbink). 
dionale, les femmes me'en empêchent les hommes d'observer le processus de fabrication de la poterie, parce que leur regard "rendra les objets fragiles et sans valeur". Tous les autres objets des Me'en, y compris les parures féminines, sont produits par les hommes.

\section{LA NATURE de LA CULTURe MATÉRIELLE des ME'EN ET SON ÉVALUATION}

Presque toutes les familles me'en utilisent la même catégorie d'objets. Il n'y a pas de grandes différences dans le nombre de leurs possessions matérielles. De plus, les Me'en n'opèrent pas une stricte distinction entre les outils quotidiens, pour préparer l'alimentation, et les objets décoratifs. La dénomination générale pour tous ces objets est á'a : "objets", "choses". Ils n'utilisent pas de mot pour "l'art" ou pour les "objets artistiques". Aussi les objets rituels, comme les bâtons à feu qui sont nécessaires pour les rites annuels liés à la récolte du maïs ou du sorgho, se classent-ils ensemble avec les calebasses et les bassins qu'on emploie pour préparer les aliments quotidiens. On observe, cependant, une certaine distinction entre les artefacts selon l'âge, le sexe et le statut social (par exemple marié ou non-marié) du possesseur : par exemple, les jeunes hommes ont toujours besoin de parures spécifiques, comme les chaînes de cuir des vaches ou les grains ou perles en verre, des bracelets en cuivre, des pendants d'oreilles en fer ou en cuivre, et un couteau personnel doté d'une poignée en ivoire ou en corne de buffle. Les jeunes filles adolescentes demandent elles aussi des parures féminines comme des pendants d'oreilles, des chaînes de perles de verre coloré, des bracelets pour les bras et les jambes, ainsi qu'un pagne de grains (nommée daafa, vorr ci-dessous), un objet très cher dont l'assemblage nécessite de nombreuses années. Les jeunes filles s'efforcent aussi d'obtenir le plus vite possible leurs propres objets de ménage comme des paniers, des bassins et des coupes de bois (photos 1 et 4), de bonne qualité, bien faits et durs. Les femmes mariées et chargées de famille ont besoin non seulement de tout cela, mais aussi de la série entière des calebasses me'en (il y en a au moins dix types), de paniers, de cuillères en bois, de poteries, et bien sûr de vêtements (on demande maintenant des pièces importées). Toute femme mariée doit avoir complété son daafa, la grande bande de perles de verre coloré qu'elles portent sur les hanches - objet qui signifie la fertilité et l'abondance (photo 2). Les hommes mariés et les aînés veulent se présenter en public munis de l'appui-nuque ou du siège en bois dur nommé chakam (photo 3), et d'une tabatière, et - quand il s'agit d'un aîné de clan - d'une lance cérémoniale (nommée jaut), un objet très recherché.

À travers ces préférences marquées selon l'âge, le sexe et le statut matrimonial, on peut constater les critères fondamentaux -bien que très limités - qui servent à valider les objets et leur esthétique. Par exemple, les jeunes gens désirent, comme tous ceux de leur âge d'ailleurs, capter l'attention du sexe opposé et se distinguer comme des personnes attirantes et capables. Sans doute les jeunes aussi bien que les adultes se réfèrent-ils aux notions de beauté ou, dans ce cas, d'aptitude ou de qualité et de talent (en produisant ou en choisissant telle forme ou telle autre). Dans le cas des jeunes, on perçoit facilement le langage de l'esthétique du corps. Chez les Me'en, on prend en compte l'apparence globale d'une personne : le nombre et la nature des parures per- 
sonnelles, mais aussi l'apparence physique (avec les scarifications, les cheveux rougis à l'ocre, la taille, la couleur de la peau rouge préférée au noir, comme ailleurs en Éthiopie), et aussi le style et le talent pour danser de manière gracieuse et pour chanter de façon originale.

Après le mariage, lors des débuts de la vie de famille, les besoins et les sensibilités changent évidemment. On ne voit pas de femmes ou d'hommes mariés utiliser les parures des jeunes, mais les hommes portent d'autres parures comme les petits trophées d'animaux tués à la chasse (par exemple des bandes de la peau des singes guré$z a$, ou de léopard), ou bien des bracelets et des colliers en matériaux divers.

De plus en plus, on se met à produire et à collectionner des objets domestiques, comme des outils, des paniers, des pots, des armes (de préférence un fusil ou une arme automatique), ainsi que le daafa et l'appui-nuque comme marques du statut de l'adulte. L'aspect "fonctionnel" des objets quotidiens va dominer en dépit de l'esthétique du corps, et cela veut dire que l'on estime les objets pour leur qualité, leur durabilité, leur efficacité, et peut-être leur couleur et leur forme. Comme pour les objets de parure, toutefois, quelques-uns de ces objets durables (en fer ou en bois, comme les lances rituelles, les appuis-nuque, les poignards travaillés avec soin) peuvent accumuler de la valeur à travers le temps. Les Me'en n'oublient pas l'histoire individuelle de ces objets : c'est une dimension qui, pour n'être pas directement visible, n'en est pas moins importante.

Dans le domaine de la production des objets décoratifs tout autant qu'utilitaires et cérémoniels, on peut certainement reconnaître un «style me'en». Les Me'en, ainsi que leurs voisins (des groupes dizi, bench ou amhara), me le soulignaient fréquemment. Par exemple, aucun Amhara, Bench ou Dizi ne se fera voir en public ou en privé avec une petite chaise/appui-nuque chakam, ou avec des sandales de cuir de buffle (chaich), ou avec des bracelets de cuir (laka). Et ceci malgré le fait que maints objets de la tradition me'en aient été adoptés par d'autres groupes en raison de leur circulation. On dit aussi que les Dizi ou les Bench ne sont pas capables de produire des calebasses, des pièces de bois, des poignards, etc., de qualité : on doit les acheter chez les Me'en. Mais en dépit de ce stéréotype et de ce style soi-disant "typiquement"

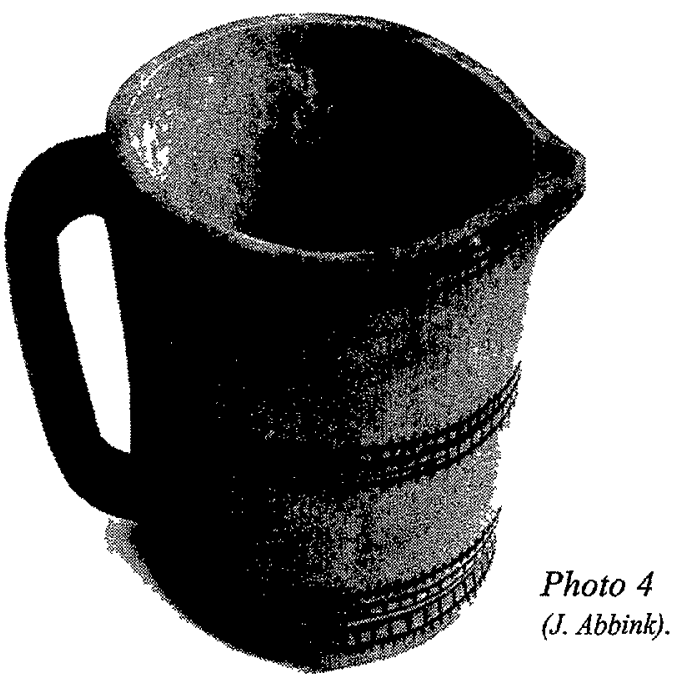
me'en, de nombreuses personnes appartenant à ces ethnies Bench et Dizi - et même quelques Amhara traversent les "frontières ethniques" et apprennent ou imitent des techniques, des motifs de décoration, ou encore certains types d'objets des Me'en. Ce phénomène d'échange entre ethnies est lui-même des plus intéressants à étudier. 


\section{LA PRODUCTION TECHNIQUE DES ARTEFACTS ME'EN}

Le nombre d'objets me'en est assez limité, ce qui correspond peut-être au nıveau technologique et matériel du mode de vie des Me'en et des échanges commerciaux de produits importés (devenus maintenant assez répandus dans le reste de l'Afrique).

On peut néanmoins distinguer plusieurs catégories chez les Me'en (ABBINK 1992) : les objets de ménage, les outils agricoles et autres, les armes, les objets décoratıfs, les objets indiquant le statut personnel et les objets cérémoniels. Il faut souligner que les Me'en sont autosuffisants dans la production de tous ces objets, et qu'en fait 1ls ne connaissent pas de relations de dépendance avec d'autres groupes (bien que les marchands des villages aient créé de nouveaux besoins matériels). Pour nous, membres de sociétés industrielles, il est difficile d'imaginer le sentiment d'autarcie et la relation étroite avec l'environnement physique qui entraînent ce défi quotidien et saisonnier de transformer les matériaux de la nature en produits et de créer les moyens de production et de nourriture. De plus, il n'y a pas de véritable spécialisation artisanale, et il manque donc une classe d'artisans. Il y a quelques forgerons (urit), mais ceux-ci ont apprns le travail auprès des forgerons des Dizi, ou sont descendants d'immigrants. Les Dizi ou les Bench ont des "castes" spécialisées de potiers et de forgerons (comme la plupart des ethnies de l'Éthiopie traditionnelle).

Les matériaux que les Me'en utilisent sont diverses sortes de bois, de l'argile, du fer, des courges, des espèces de chaume et de liber, des peaux d'animaux domestiques ou sauvages, du cuivre, des pièces de monnaie anciennes en argent (de l'ère de Haile Selassie), de l'aluminium des vieilles bouilloires, ou du métal des douilles. Bien qu'il y ait des différences individuelles qu'on reconnaît comme telles, presque tout le monde chez les Me'en sait travailler ces matériaux et en tirer les objets et les autres nécessités matérielles de la vie, ce qui veut dire que la tradition des artefacts est un art "démocratique". Les enfants l'observent dès leur jeunesse, et l'apprennent en imitant les parents.

\section{QUELQUES EXEMPLES}

Bien que l'esthétique des Me'en se révèle dans beaucoup de domaines de leur culture matérielle (y compris, surtout chez les gens du qolla, par les vêtements de cuir des femmes, les lanières décorées de cauris, les décorations du corps et du bétail), nous voudrions présenter brièvement ici, les façons de travailler de certains producteurs pour illustrer la production actuelle de quelques objets importants ${ }^{5}$.

\section{a. Bois}

Le premier processus de fabrication est celui des objets en bois. J'avais choisi un homme connu pour son habileté mais qui n'était pas un artisan spécialisé : il disait qu'il était aussi, et en premier lieu, un paysan ; en effet comme presque tout Me'en adulte, il laboure les champs pour sa famille (de six personnes). La différence est que cet homme produit des objets pour la vente. Sa femme n'était pas une spécialiste non plus, bien qu'elle sache-comme toute femme me'en - produire de la céramique comme les grands plats à cuire et les grand pots pour bouillir et préparer la nourriture.

${ }^{5}$ Volr ABBnK 1992 pour l'inventare d'autres objets me'en 

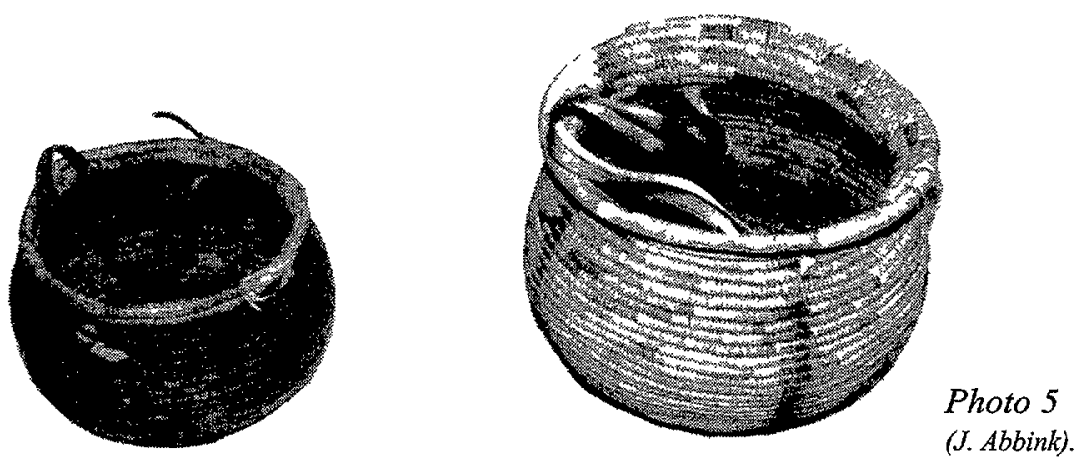

Ce fabricant, nommé Bogine Shala, commença à vendre des objets "par hasard", lorsque quelques visiteurs d'une autre région lui demandèrent de leur donner quelques-uns de ses objets en bois et de ses paniers. Alors que la vente des objets lui assurait un faible revenu, Bogine estimait ne pas pouvoir vivre de ses ventes, même si le prix en avait été doublé. Ceci me fut confirmé d'ailleurs par les autres fabricants.

Bogine disait qu'il savait faire les objets en bois, les calebasses, etc., simplement parce qu'il avait toujours observé son père le faire. Il n'y a rien de secret, de difficile ou de rituel dans la production des outils et des produits matériels : les objets ne sont pas connotés d'aspects surnaturels ou magiques, et il n'y a pas de dimension religieuse ou occulte qui les mettrait à l'écart de la vie quotidienne. L'aspect technique et pragmatique est toujours dominant. Il n'y a pas de tabous qu'on devrait respecter en produisant ou en utilisant les artefacts (sauf pour les grands plats à cuire [retech], produits pas les femmes, voir plus haut).

La production d'une coupe en bois demande seulement quelques quatre ou cinq heures. Tout d'abord, il faut trouver une espèce d'arbre d'une qualité convenable, par exemple le Cordia africana Lam. On n'utilise que la hache et la machette (banga). Un petit couteau est nécessaire pour la finition.

Les Me'en Bodi, dans la vallée des rivières Omo et Shorum (les Mela, Chirim, Shua et Dabashinto), utilisent les feuilles de l'arbre $k$ 'araich (Lippia grandifolia Hochst.) pour polir le bois. Mais dans les régions des Me'en Tishana, on ne trouve pas cet arbre. En général, les Me'en de la vallée sont considérés comme plus doués pour la production d'objets attractifs : ils sont les "maitres". En effet, leurs objets - surtout les appuis-nuque, les paniers, les coupes de bois, les parures personnelles et celles du bétail, les couteaux - sont faits avec plus de soin et plus d'attention pour les détails de décoration, la couleur, le choix des matériaux, ainsi que pour la forme (avec plus de variations individuelles au cours de l'exécution de l'objet). Ainsi, les objets des hommes de la vallée sont unanimement préférés. Un très bel exemple en est fourni par la coupe de la photo 4 , faite par un homme du qolla.

\section{b. Paniers}

Les Me'en possèdent seulement un petit nombre de paniers et de corbeilles: le zarzarach pour cribler la bière de sorgho, le woshi pour sécher les grains, les poivres, etc., et deux sortes de paniers (garju) pour offrir ou servir de garde-manger. On n'y applique pas de décorations ou de couleurs, comme le font les Oromo ou les Adaré. 
Le garju rond est un produit des Me'en de la vallée, parce qu'on ne trouve pas de matériau comme les feuilles du palmier Hyphaene thebaica dans les hautes terres de la wäyna däga (photo 5). On ne trouve pas cet objet chez les voisins des Me'en comme les Dizi, les Aari ou les Anywak et même pas chez les Me'en de la wáyna däga qui les achètent à leurs frères de la vallée.

Le panier woshi (photo 6) est un produit qu'on trouve chez les Me'en de la wäyna däga, mais pas chez les hommes de la vallée. L'objet figuré est d'une forme spécifique, faite avec la technique des rouleaux ${ }^{6}$ que l'on ne voit habituellement que chez les garju, parmi les Me'en de la basse vallée : en général, les woshi des Me'en de la zone wäyna däga utilisent la technique «chequer-weave». L'objet figuré est plus petıt que l'exemplaire normal, et il est aussi fait de fibres végétales différentes. Le fabricant l'a fait en un jour en utilisant les branches du buisson ket-te-koroy (Rhamnus prinoides L'Hérit. = bois de nerprun) et l'écorce de la plante bants'alach (Periploca linearlfolie A. Rich.). Le problème de fabrication est ici de réaliser une vraie assiette : ronde, légèrement convexe et souple. Bien que le travail paraisse assez simple, personne dans le village ne pouvait le faire comme Bogine.

\section{c. Calebasses}

Les Me'en ont besoin de calebasses de formes et de fonctions très différentes. Les calebasses sont faites des fruits d'une courge (Lagenaria siceraria ou Lagenaria vulgaris). En effet, en vivant parmi les Me'en, on se rend compte que les calebasses constituent le type d'objet domestique le plus répandu et le plus important. On observe beaucoup de modèles de différentes grandeurs. La photo 7 nous en montre quelques types. La calebasse est aussi l'un des rares objets présents chez les Me'en qui soit décoré avec des figures géométriques (comme les peignes, les bracelets de cuivre, les étuis de couteau, et - rarement - les appuis-nuque). Seuls les hommes fabriquent ces outils; les femmes ne touchent pas à ce travail.

Chaque famille me'en plante les courges dans le jardin près de la maison. Pendant la période de croissance, on peut bander les fruits pour influencer leur forme. Par exemple, pour le type de calebasse $b^{\prime}$ ogol, il faut entailler le milieu du fruit. Pour une $k^{\prime}$ ada (une forme de "bouteille"), au contraire, on doit créer un cou éloigné en bandant la partie supérieure.

Les calebasses sont décorées avec des motifs géométriques - triangles, lignes et cercles non-figuratifs - et sont gravées à l'extérieur avec un pic en fer (mudá). Ensuite, on frotte du charbon dans le dessin pour lui donner sa couleur noire. Ces dessins, qui pourraient ressembler à des chemins, à un serpent, à un grenier, ou à un groupe de maisons, $n$ 'ont pas une telle signification, directe ou indirecte, pour les Me'en. Mes questions posées aux fabricants et utilisateurs afin d'y déceler un symbolisme sous-jacent restèrent sans réponse. On trouve les mêmes motifs dans le daafa, le pagne de grains colorés des femmes. L'origine de ces motifs - assez communs chez les peuples voisins, Surma, Mursi et Dizi - n'est pas claire.

La fabrication d'une calebasse semble encore plus simple que celle d'un objet de bois ou d'un panier comme nous l'avons vu plus haut. Mais malgré le fait que la plu-

\footnotetext{
${ }^{6}$ En anglass "coll"
} 
part des hommes me'en en produisent, il faut savoir s'y prendre afin de ne pas gâter le nombre limité des fruits de la courge que l'on récolte chaque année (surtout au mois de septembre). Ainsi, on voit des spécialistes qui produisent ces objets d'une façon plus ou moins "commerciale".

La fabrication des calebasses s'opère ainsi : on fait sécher les fruits frais ( $k$ 'ajach) de la courge pendant une semaine ; les graines et la pulpe à l'intérieur du fruit sont détachées ou coupées avec un pic, ma1s il est alors impossible de les enlever. Cela sera possible après quelques jours, voire après une semaine. On garde les graines pour les sécher et les planter la saison prochaine. Les fruits secs et nettoyés sont ensuite coupés très doucement selon le modèle de l'objet que l'on veut produire, par exemple au milieu pour faire des tasses à café ou à bière (sholu) ou des calebasses pour les aliments, ou encore seulement la partie supérieure, pour faire une ouverture susceptible d'être bouchée, etc. Des mains inexpérimentées détruiraient facilement le fruit entier.

Quand on a modelé la forme élémentaire de la calebasse, on laisse l'objet dehors, couvert de boue, de sable ou d'herbes. Quelques semaines plus tard, on le reprend pour le finir et le polir. On enlève les restes de la "peau extérieure" du fruit avec un couteau, on coupe les bords une nouvelle fois, et on nettoie l'extérieur avec du sable et des feuilles. Après cela, l'objet est encore laissé de côté durant quelques jours.

Le travail final est celui de la décoration avec les dessins géométriques, déjà mentionné. Quand la calebasse est destinée à la vente, on ne lui applique pas de dessins : c'est l'acheteur lui-même qui le fera. Bien que les calebasses soient simples et bon marché, on les soigne assez bien : quand une calebasse est cassée par accident, on la tépare patiemment et très adroitement avec des fibres végétales. Par un polissage fréquent et un nettoyage à l'huile de ricin, on peut aussi donner une certaine patine à la calebasse. Ainsi l'identité spécifique et la beauté de l'objet se trouvent renforcées.

\section{FONCTION ET FORME DES ARTEFACTS ME'EN}

Nous avons brièvement traité de trois types d'objets situés dans la sphère domestique des Me'en, dans le but de réfléchir sur "l'art du quotidien"7. Pour cette raison, nous n'avons offert qu'une analyse préliminaire des objets décoratifs comme l'appui-nuque ou le daafa. Les trois catégories d'objets ont été discutées pour explorer des éléments qui pourraient définir une tradition esthétique à travers des objets simples du point de vue technologique. Ces trois catégories d'objets 'n'ont pas de valeur rituelle ou cérémonielle évidente, ni une valeur pécuniaire ou spécifiquement esthétique pour les Me'en eux-mêmes. Mais tous les objets bien faits, comme les daafa ou les appuis-nuque, sont appelés en langue me'en ande-she ' $i$, ce qui signifie des objets de "qualité", de

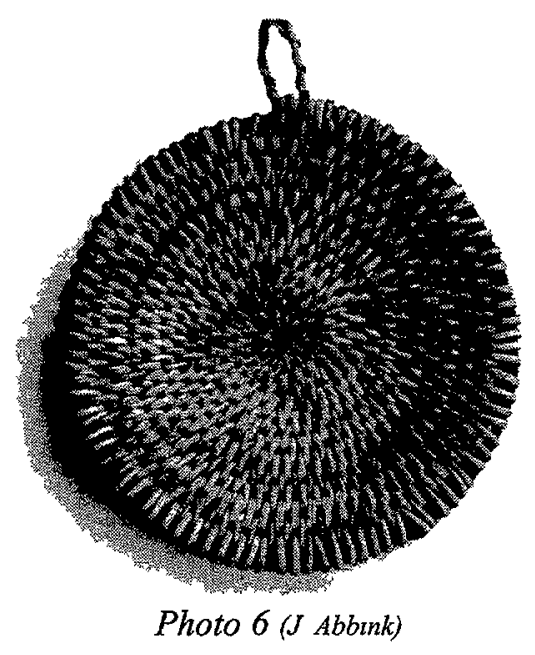


"fonctionnalité" ou "d'aptitude". Le mot she'i en langue me'en regroupe l'ensemble des notions de "bien", "dur", "correct", "apte", ce qui veut dire qu'à propos des objets matériels, la qualité d'être she' $i$, se trouve dans cette combinaison entre une "aptitude morphologique" de l'objet et son "efficacité fonctionnelle". Ces caractéristiques s'expriment sous l'apparence d'une "attraction naturelle" d'un objet, ou bien, en employant nos termes, peut-être de sa beauté.

Comme disent les Me'en, un objet beau et bien fait se caractérise par un choix réfléchi de matériaux durables, par sa fonctionnalité, par un dessin et une exécution réalisés par un expert. Tout cela n'est guère étonnant pour des gens issus d'une culture technologique très développée, mais on ne se rend pas compte du fait que l'application de techniques et d'outils rudimentaires à des matériaux naturels souvent réfractaires est un défi quotidien, qui doit être résolu par tout homme ou femme me'en. Il faut toujours opérer une série de choix apparemment insignifiants mais mesurés et adroits pour produire un résultat acceptable.

Beaucoup d'autres objets des Me'en montrent une exécution stylistıque soignée qui va au delà d'une simple fonctionnalité pratique. Cette apparence esthétique est plus claire dans les objets personnels comme les appuis-nuque (SIEBER $1980: 108$ ), les calices de bois ou les petites tabatières, très caractéristiques des hommes me'en de la région du qolla. Ces objets personnels ne sont pas achetés ou vendus de façon commerciale : on les donne comme cadeau, on les échange avec des amis ou des parents pour entretenir une relation de longue durée. La valeur de ces objets est fondée sur la réputation de leurs fabricants, de leurs possesseurs et de leur propre "trajet" (SIEBER $1980: 108,125)$. Ainsi leur valeur augmente au cours du temps et ne peut pas être exprimée en termes monétaires. Par exemple, la photo 3 montre un appui-nuque (chakam) assez rare, qui était la possession d'un chef local d'un des douze clans "originels" des Tishana-Me'en. L'objet a été fait par son père, décédé en 1960 environ. Le bois (en Me'en : tamshit; je n'ai pas pu déterminer le nom scientifique) est d'une espèce dure de la région du qolla. L'objet est sans doute précieux, mais on ne parlait jamais de son "prix" ou de sa valeur d'échange. Plusieurs de ces objets sont admirés par les Me'en non pas pour leur "fonctionnalité" etc., mais plutôt pour leur aptitude de forme, de qualité, de couleur, et aussi en raison de l'histoire de leur circulation.

Il va sans dire que ces objets, plus ou moins "détachés" du domaine quotidien du foyer et de la vie familiale, sont les plus pertinents pour des observateurs extérieurs comme nous. Certes cela constitue peut-être un nouveau biais, presque inévitable, qui résulte d'une esthétique plus ou moins universelle qui met l'accent sur la symétrie, la clarté des formes et de l'espace, l'autonomie de l'artefact ou bien encore son apparence d'unité et d'harmonie. Mais cela souligne également le fait qu'une tradition simple, non-technologique et non-spécialiste comme celle des Me'en, montre une sensibilité qui ressemble à la nôtre dans ses formes élémentaires.

${ }^{7}$ Nous espérons présenter une étude plus étendue de la culture maténelle des Me'en dans un autre ouvrage 


\section{ConCLUSION}

La culture matérielle des Me'en est en premier lieu le produit d'un groupe non-hiérarchique et égalitaire. L'absence de "chefs" et d'hommes puissants et riches qui auraient pu jouer le rôle de commanditaires de l'art a évité la formation d'une classe d'artisans (comme elle existait, par exemple, parmi les chefferies et royaumes d'Afrique centrale et occidentale) et la constitution de l'art comme domaine autonome. Ceci a engendré une sorte "d'égalitarisme de sentiment et de sensibilité artistique", ne privilégiant aucun objet comme étant un accomplissement spécial ou unique. Les conventions me'en de production et d'usage se manifestent surtout dans la fonctionnalité et l'efficacité des objets du point de vue des usagers, tandis que la propre "valeur" d'un objet particulier est plutôt liée à son histoire et à son trajet social -mais cela ne vise pas toutes les catégories d'objets.

En parlant de l'égalitarisme des sentiments engendrés par ces objets me'en, nous voulons souligner le fait que la "force" des artefacts, la manifestation d'une sensibilité ou d'un sentiment par les Me'en à leur égard, est plus ou moins uniforme. Le monde des objets n'est pas compris de façon systématique comme un domaine où s'expriment les différences personnelle ou même les valeurs esthétiques et culturelles. Nous avons dit que presque tout Me'en adulte est capable de produire des objets, et que l'on ne devient pas un expert ou un homme paré de vertus spécifiques à cause de la production artisanale.

La production et la nature des objets me'en rendent assez prévisibles la nature et la quantité des objets que l'on va pouvoir produire, acheter ou obtenir par échange. Il n'y a pas une production d'objets détachée des contextes de la vie quotidienne. Les objets décoratifs et rituels appartiennent également à ce domaine. Sans nier l'influence croissante des dynamiques externes et internes chez les Me'en, on note que la culture matérielle des Me'en est encore dominée par des notions de tradition, bien qu'interprétées de façon pragmatique. On a toujours tiré profit de tous les matériaux disponibles, y compris les débris de fer (de machines, de voitures), d'aluminium, etc. Il n'est peutêtre pas difficile de prévoir ce qui se passera en cas d'accélérations des contacts commerciaux entre les Me'en et la société éthiopienne en général : adoption rapide de nouveaux objets domestiques, d'armes (automatiques), d'outils, de vêtements, etc. Mais très vraisemblablement aussi, on assistera à la conservation des objets rituels, des

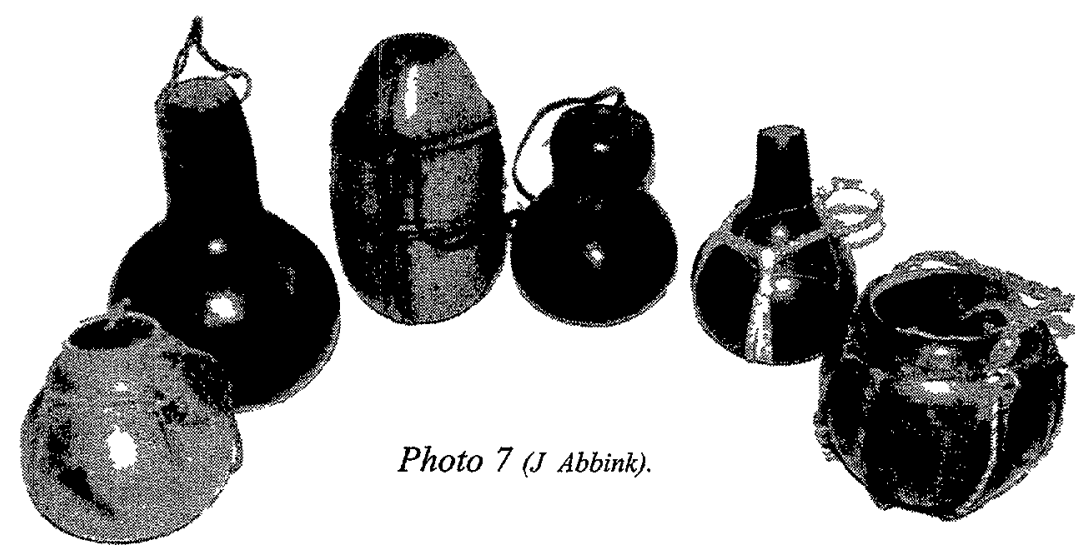


parures personnelles et des appuis-nuque, qui caractérisent le mieux l'identité des Me'en. Dans le contexte actuel de mondialisation, on peut même imaginer l'émergence d'une production plus commerciale de ces objets "typiques" (appuis-nuque, bracelets, colliers de cuir, calebasses décorées, coupes de bois, couteaux...) pour les touristes et les voyageurs, parce qu'il y a un marché croissant à Addis Abeba pour les objets "ethniques" ou "tribaux", qui est de plus en plus fréquenté par les marchands étrangers. La commercialisation et l'importation de nouveaux produits offriront sans doute de nouvelles possibilités aux Me'en, mais elles vont introduire aussi une dépendance socio-économique et un abandon des conventions de la production matérielle des Me'en. Cependant, dans ce cas, il serait intéressant de voir si les conceptions me'en de beauté implicite, d'aptitude et d'efficacité des objets - 1'unité sous-jacente de forme et de fonction - disparaitront elles aussi, ou bien si elles s'attacheront à certains objets "résistants" que l'on considérera comme les éléments de l'identité ethnique me'en.

\section{BIBLIOGRAPHIE}

ABBINK (J.), 1990 : Tribal formation on the Ethiopian fringe : toward a history of the «Tishana». Northeast African Studies 12, 1, p. 21-42.

ABBINK (J.), 1992: The Me'en of Southwestern Käfa. Material Culture of an Ethiopian Shifting-cultivator people. Addis Ababa: Institute of Ethiopian Studies.

AMBORN (H.), 1990: Differenzierung und Integration. Vergleichende Untersuchungen zu Spezialisten und Handwerkern in südäthiopischen Agrargesellschaften. Munich: Trickster Verlag.

DECARBo (E.A.), 1977 : Artistry among Kasem speaking Peoples of Northern Ghana. Bloomington: Indiana University, Department of Anthropology (Thèse de Doctorat).

FalgeYrettes (C.), 1989 : Supports de Rêves. Paris: Fondation Dapper.

BELL (A.), 1991 : The technology of enchantment and the enchantment of technology. In: COOTE (J.) \& SHELTON (A.) eds., Anthropology, Art and Aesthetics, p. 40-63. Oxford: Clarendon Press.

KLOPPER (S.), 1993 : The carver in Africa : individually acclaimed artist or anonymous artisan? Social Dynamics 19, 1, p. 39-51.

SIEBER (R.), 1980: African Furniture and Household Objects. New York: American Foundation of Arts / Bloomington: Indiana University Press.

TORNAY (S.), 1975 : La culture matérielle des Nyangatom. In: Ethiopie d'aujourd'hui. La Terre et les Hommes, p. 45-52. Paris: Musée de l'Homme.

VON GAGERN (A.) et al., 1974. Ostafrika - Figur und Ornament. Hamburg: Museum für Völkerkunde. 